\title{
Clindamicina como terapia adyuvante en el síndrome de piel escaldada estafilocócica
}

\section{Clindamycin as adjuvant therapy in staphilococcal skin scalded syndrome}

\author{
F.J. Gil Sáenz ${ }^{1}$, M. Herranz Aguirre ${ }^{2}$, G. Durán Urdániz ${ }^{1}$, L. Zandueta Pascual ${ }^{1}$, \\ J. Gimeno Ballester ${ }^{1}$, E. Bernaola Iturbe ${ }^{2}$
}

\section{RESUMEN}

El síndrome de piel escaldada estafilocócica (SPEE) es una enfermedad dermatológica causada por exotoxinas producidas por $S$. aureus. La enfermedad cursa como un exantema cutáneo doloroso que culmina con el desprendimiento de la dermis superficial. El tratamiento habitual es antibioterapia con penicilinas resistentes a betalactamasas. Presentamos el caso de una paciente con SPEE que presentó una evolución inicial tórpida a pesar del tratamiento antibiótico y que tras la introducción de clindamicina intravenosa experimentó una mejoría muy importante. La preocupación por el progresivo aumento de estafilococos aureus meticilín resistentes (SAMR) y el mecanismo fisiopatológico de esta enfermedad hacen de los antibióticos bacteriostáticos de espectro antiestafilocócico, como la clindamicina, firmes candidatos para ser considerados como arsenal terapéutico de primera línea en el tratamiento del SPEE.

Palabras clave. Síndrome de piel escaldada. Estafilococcus aureus. Clindamicina.

\begin{abstract}
Staphylococcal scalded skin syndrome (SSSS) is a dermatologic disease caused by exotoxins produced by Staphylococcus aureus. The disease presents as a painful cutaneous rash that culminates with the detachment of the superficial dermis. The usual treatment is antibiotics with beta-lactamase resistant penicillin. We report the case of a patient who presented with SSSS with initial torpid evolution despite antibiotic treatment and after the introduction of intravenous clindamycin experienced a very significant improvement. Concerns about the increase of methicillin resistant Staphylococcus aureus (MRSA) and the pathophysiology of this disease make bacteriostatic spectrum antistaphylococcal antibiotics, such as clindamycin, strong candidates for consideration as a first-line therapeutic arsenal for the treatment of SSSS.
\end{abstract}

Key words. Staphylococcal skin syndrome. Staphylococcus aureus. Clindamycin.
1. Servicio de Pediatría. Hospital García Orcoyen. Estella.Navarra.España.

2. Servicio de Pediatría. Complejo Hospitalario de Navarra. Pamplona. Navarra. España.

Recepción: 19 de junio de 2014

Aceptación provisional: 30 de septiembre de 2014

Aceptación definitiva: 2 de octubre de 2014

\section{Correspondencia:}

F.J. Gil Sáenz

Hospital García Orcoyen

Servicio de Pediatría

C/Santa Soria 22

31200 Estella (España).

E-mail: fj.gil.saenz@cfnavarra.es 


\section{INTRODUCCIÓN}

El síndrome de piel escaldada estafilocócica (SPEE), es una enfermedad dermatológica causada por algunas bacterias del género estafilococo, sobre todo cepas de $S$. aureus. La enfermedad se origina por ac- ción de las exotoxinas epidermolíticas A y B (ETA y ETB) liberadas por el estafilococo, que rompen la desmogleína-1 (DMG1) que mantiene unidos los estratos granulosos y espinosos de la dermis (Fig. 1) ${ }^{1}$, originando la formación de ampollas por desprendimiento intraepidérmico ${ }^{1,2}$.

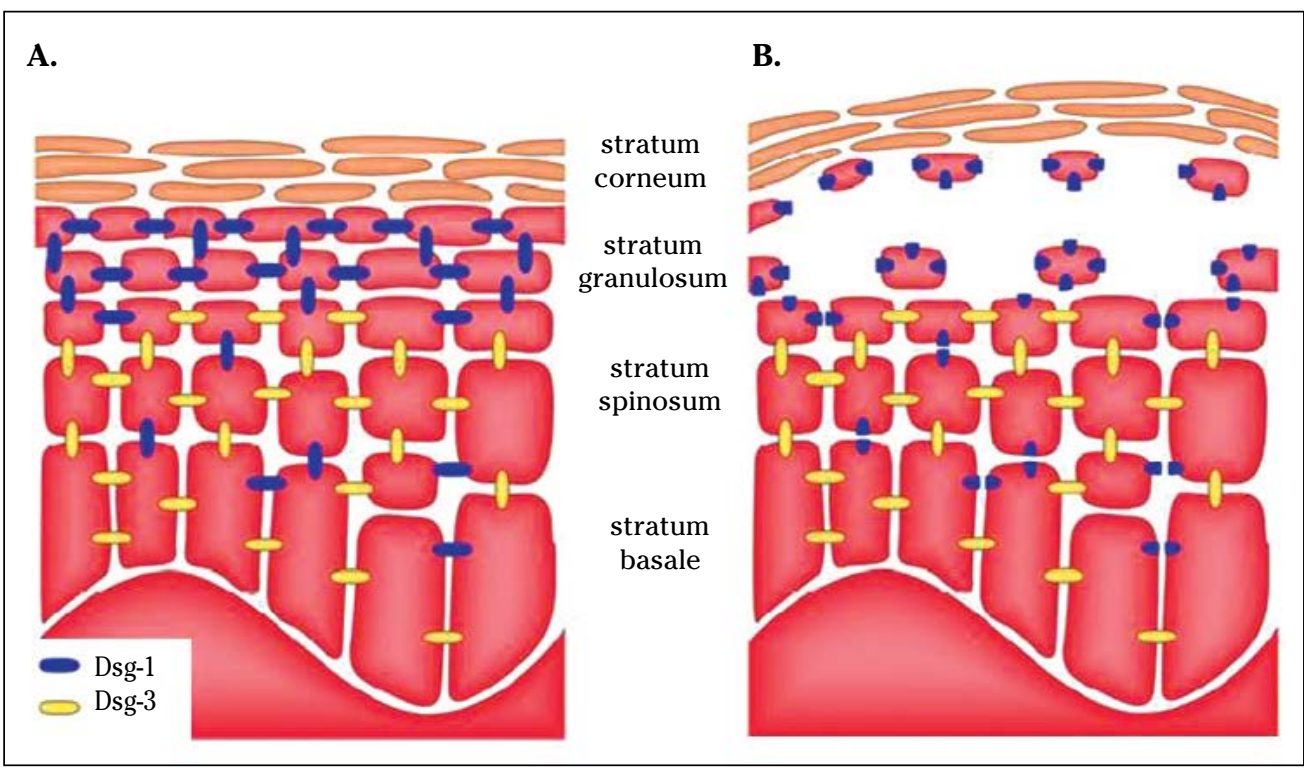

Figura 1. Distribución de la DMG1 en los distintos estratos de la dermis (izda) y mecanismo de desprendimiento intraepidérmico en el SPEE (dcha).

Es una enfermedad infrecuente, con una incidencia de 0,13 a 0,56 casos/millón, siendo la mediana de edad de dos años y no existiendo diferencias significativas a nivel de sexo $^{3,4}$.

El cuadro clínico suele manifestarse con fiebre, malestar general y lesiones cutáneas eritematosas que suelen localizarse sobre todo en cara, cuello, axilas y región perineal $^{2}$. Posteriormente se forman ampollas en las zonas eritematosas y el signo de Nikolsky suele ser positivo. Las lesiones suelen curar sin dejar cicatriz ${ }^{2}$.

La gravedad del síndrome es muy variable siendo las tasas de mortalidad descritas del 4 al $11 \%^{2,3}$, aunque en adultos la mortalidad puede ser del $40 \%^{3}$.
En su diagnóstico diferencial hay que tener en cuenta la necrólisis epidérmica tóxica (NET), así como otras enfermedades dermatológicas ${ }^{5}$.

El tratamiento habitual es antibioterapia con penicilinas resistentes a betalactamasas ${ }^{2}$, salvo sospecha de estafilococos meticilín resistentes (SAMR) ${ }^{6}$. Presentamos el caso de una paciente que experimentó un cambio llamativo en la evolución de su enfermedad tras la adicción de clindamicina al tratamiento.

\section{CASO CLÍNICO}

Presentamos el caso de una niña de 4 años que consultó por un exantema eritrósico y doloroso en región cervical de 12 horas de evolu- 
ción. La paciente tenía malestar general, no presentaba fiebre, síntomas respiratorios ni otros síntomas añadidos. Había sido valorada hacía 6 horas como un posible prúrigo, habiendo iniciado tratamiento con corticoide y antihistamínico por vía sistémica. A la exploración física presentaba eritema cutáneo en cuello, axilas, tórax y nalgas, con áreas de despegamiento cutáneo en zona axilar y cervical y alguna lesión costrosa amarillenta en región cervical (Fig. 2). También mostraba eritema e inflamación palpebral y conjuntival, siendo el resto de la exploración física normal. Las constantes de frecuencia cardiaca, respiratoria y presión arterial fueron normales. Se realizó analítica de sangre que mostraba leucocitosis (12.300 leucocitos $/ \mu \mathrm{L}$, fórmula normal) sin elevación de reactantes de fase aguda.

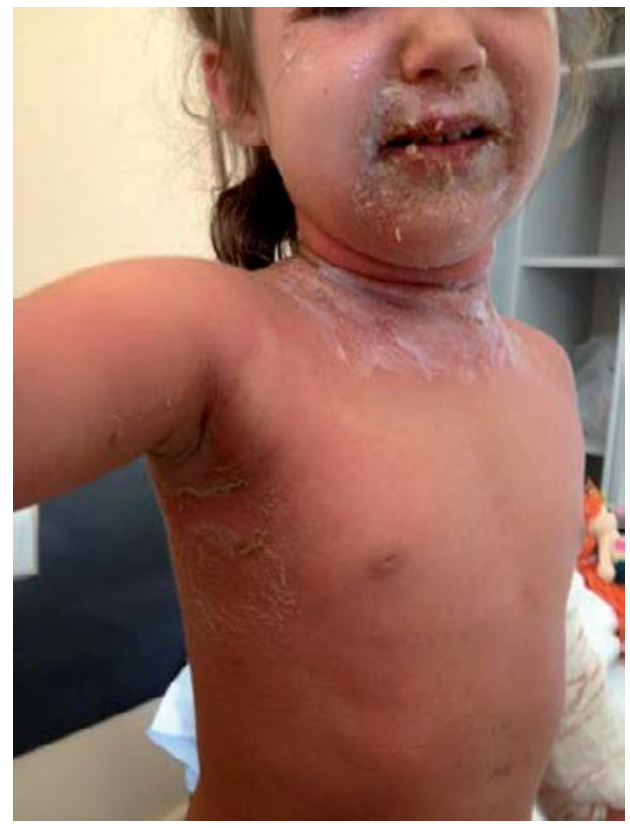

Figura 2. Eritrodermia cutánea y lesiones periorales y axilares en distintos estadios.

Con diagnóstico presuntivo de SPEE ingresó e inició tratamiento con cloxacilina i.v, mupirocina tópica, antihistamínico i.v, fluidoterapia i.v, y analgesia. La evolución fue inicialmente desfavorable, con generalización de las lesiones y extensión de la eritrodermia. Al tercer día se asocia empíricamente al tratamiento clindamicina i.v, constatando a las 24 horas una evolución muy favorable de las lesiones. Posteriormente el hemocultivo fue informado como negativo $\mathrm{y}$ el cultivo de frotis cutáneo y nasal fue positivo para $S$. aureus (resistente a penicilina, sensible a cloxacilina, amoxicilna-clavulánico y clindamicina). Tras seis días de ingreso con tratamiento parenteral se decide alta para continuar el tratamiento antibiótico de manera ambulatoria.

\section{DISCUSIÓN}

El SPEE es una entidad clínica rara que se manifiesta fundamentalmente en la infancia. El espectro de esta enfermedad abarca desde formas generalizadas (enfermedad de Ritter en neonatos) hasta formas localizadas de impétigo bulloso ${ }^{4}$. Tiene su máxima incidencia en niños menores de 5 años $^{2}$, que suelen ser previamente sanos. Sin embargo, en adultos el desarrollo de esta enfermedad suele ocurrir en personas con factores predisponentes, como la falta de aclaramiento renal de exotoxinas (insuficiencia renal, consumo crónico de AINE...) y la inmunosupresión (VIH, pacientes oncológicos, inmunodeficiencias, abuso de alcohol y drogas, diabetes... $)^{2}$.

La fisiopatología se fundamenta en la acción de las toxinas exfoliativas ETA y ETB ${ }^{1,2}$, proteasas que producen la hidrólisis de la DMG1. La ruptura de la DMG1 produce un deterioro en la adhesión de los queratinocitos, fundamentalmente en el estrato granuloso, dado que a este nivel no existen otras moléculas de adhesión que sí están presentes en sustratos más profundos (Fig. 1) ${ }^{1}$.

El diagnóstico de esta entidad se fundamenta en la clínica, la microbiología y la histología ${ }^{2}$. Clínicamente se caracteriza por la existencia de un cuadro de eritrodermia con lesiones vesiculosas, siendo muy frecuente la presencia del signo de Nykolsky ${ }^{6}$. Desde el punto de vista microbiológico es necesaria la evidencia en algún cultivo de S. aureus productor de exfoliatinas ${ }^{2}$. Los cultivos de las lesiones ampollosas así como los hemocultivos suelen ser negativos para $S$. aureus en los niños, mientras que los cultivos nasofaríngeos suelen ser positivos $^{6}$. El análisis histológico ayuda a confirmar el diagnóstico y descartar otras patologías $^{2}$.

El diagnóstico diferencial se debe hacer inicialmente con la NET, diferenciándose fundamentalmente en que el SPEE no pre- 
senta afectación de mucosas y a que el desprendimiento de la epidermis es más superficial (análisis histológico por biopsia) ${ }^{6}$.

También se incluirían en el diagnóstico diferencial otras entidades como el pénfi$\mathrm{go}^{2}$, las infecciones por enterovirus ${ }^{6}$, el Sd de Steven-Johnson ${ }^{6}$, la enfermedad injerto contra huésped ${ }^{5}$, la mastocitosis bullosa ${ }^{5}$, la reacción por drogas con eosinofilia (Sd DREES) ${ }^{6} \ldots$

El shock tóxico por estafilococo (STE) es otra enfermedad estafilocócica causada por toxinas y consiste en una enfermedad aguda que habitualmente se manifiesta como shock y fallo multiorgánico. En este caso la acción de toxinas superantígeno y enterotoxinas estafilocócicas ${ }^{7}$ produce una activación exagerada de la cascada inflamatoria que finalmente conduce al shock ${ }^{8}$. Ambas entidades tienen en su espectro formas leves que pueden ser indistinguibles salvo si se realiza el estudio genético de los gérmenes ${ }^{7}$. Courjon y col demostraron al analizar 22 pacientes diagnosticados de cuadros leves de SPEE que existían dos cuadros clínicos distintos, uno de ellos producido por exfoliatinas y otro producido por toxinas superantígenos (incluiría formas abortivas de STE y cuadros previamente denominados como fiebre escarlatina estafilocócica) ${ }^{7}$.

El manejo terapéutico del SPEE incluye antibioterapia, fluidoterapia, analgesia y cuidados de la piel ${ }^{6}$. Hay que prestar especial atención a la reposición hidroelectrolítica, puesto que estos pacientes se comportan hemodinámicamente como grandes quemados y existe riesgo de hipovolemia e hiponatremia ${ }^{6}$. La analgesia se debe realizar con paracetamol o mórficos según necesidad, evitando la administración de AINE, que podrían interferir con el aclaramiento renal de toxinas ${ }^{6}$. El uso de corticoides se desaconseja porque podría empeorar el cuadro clínico ${ }^{2}$. En caso de empeoramiento del cuadro, a pesar del tratamiento antibiótico, podría resultar de utilidad el tratamiento antitoxina con plasma fresco congelado o gammaglobulinas ${ }^{6}$.

El tratamiento antibiótico clásico para el SPEE han sido las penicilinas resistentes a betalactamasas ${ }^{2}$. Sin embargo, la preocu- pación por el incremento de cepas SAMR ${ }^{9,10}$ ha llevado a buscar alternativas, como vancomicina, clindamicina y linezolid ${ }^{9}$. La clindamicina por su capacidad de inhibir la producción de toxinas es tratamiento de primera línea en enfermedades estafilocócicas causadas por toxinas ${ }^{8-10}$. La utilidad de los tratamientos bacteriostáticos ha sido demostrada tanto en modelos animales $^{10}$ como en humanos, considerándose tratamiento de elección en el STE ${ }^{8,9}$.

En nuestro caso, la evolución de la enfermedad inicial con tratamiento betalactámico fue tórpida, con progresión de las lesiones y aumento de la extensión de la eritrodermia. Algunos autores apuntan a que en la enfermedad producida por toxinas los betalactámicos, debido a su mecanismo de acción, pueden no ser capaces de controlar la infección o incluso empeorar los síntomas (inactividad de estos antibióticos en fase estacionaria, falta de supresión de la producción de toxinas, inducción de la producción de toxinas, liberación masiva de toxinas) ${ }^{9}$. Por esto hay autores que recomiendan no usar un betalactámico en monoterapia en la SPEE, recomendando la asociación de clindamicina ${ }^{9,10}$ o linezolid ${ }^{10}$, según patrones de resistencia locales. Por otro lado los patrones de resistencia antibióticos en las series históricas de SPEE muestran una sensibilidad del $86 \%$ a cloxacilina, frente a un $52 \%$ a clindamicina, por lo que la monoterapia con clindamicina tampoco sería una buena opción ${ }^{9}$. En nuestro medio la única serie documentada no mostraba la existencia de ningún caso de SPEE asociado a SAMR ${ }^{5}$, aunque no se especificaban las resistencias a clindamicina.

En el caso de nuestra paciente, la asociación de clindamicina modificó drásticamente el curso de la enfermedad. Hoy por hoy no existe evidencia firme que avale esta actitud terapéutica ${ }^{7}$, pero dada la baja incidencia de la enfermedad ${ }^{3,4,11}$ se antoja difícil la realización de estudios que tengan una metodología y tamaño muestral adecuados para obtener un grado de evidencia elevado. Por esto algunos autores han recomendado recientemente la combinación de clindamicina y penicilina resistente a betalactamasa como tratamiento empírico en el SPEE ${ }^{9}$. 


\section{BIBLIOGRAFÍA}

1. Bukowski M, Wladyka B, Dubin G. Exfoliative toxins of Staphylococcus aureus. Toxins (Basel) 2010; 2: 1148-1165.

2. Patel GK, Finlay AY. Staphylococcal scalded skin syndrome: diagnosis and management. Am J Clin Dermatol 2003; 4: 165-175.

3. Mockenhaupt M, Idzko M, Grosber M, Schopf E, NORGaUER J. Epidemiology of staphylococcal scalded skin syndrome in Germany. J Invest Dermatol 2005; 124: 700-703.

4. Lamand V, Daumalder O, Tristan A, Casalegno JS, Meugnier H, Bes M et al. Epidemiological data of staphylococcal scalded skin syndrome in France from 1997 to 2007 and microbiological characteristics of Staphylococcus aureus associated strains. Clin Microbiol Infect 2012; 18: E514-521.

5. Nso Roca AP, Baquero-Artigao F, García-Miguel MJ, de José Gómez MI, Aracil Santos FJ, del CasTILLO MarTín F. Staphylococcal scalded skin syndrome. An Pediatr (Barc) 2008; 68: 124127.

6. HANDLER MZ, Schwartz RA. Staphylococcal scalded skin syndrome: diagnosis and ma- nagement in children and adults. J Eur Acad Dermatol Venereol 2014 20. doi: 10.1111/ jdv.12541.

7. Courjon J, Hubiche T, Phan A, Tristan A, Bès M, VANDENESCH F et al. Skin findings of Staphylococcus aureus toxin-mediated infection in relation to toxin encoding genes. Pediatr Infect Dis J 2013; 32: 727-730.

8. Lappin E, Ferguson AJ. Gram-positive toxic shock syndromes. Lancet Infect Dis 2009; 9: 281-290.

9. Braunstein I, Wanat KA, Abuabara K, McGowan KL, Yan AC, TREat JR. Antibiotic sensitivity and resistance patterns in pediatric staphylococcal scalded skin syndrome. Pediatr Dermatol 2013 23. doi: 10.1111/pde.12195

10. Stevens DL, Ma Y, Salmi DB, McIndoo E, WaLLACE RJ, BRYANT AE. Impact of antibiotics on expression of virulence-associated exotoxin genes in methicillin-sensitive and methicillin-resistant Staphylococcus aureus. J Infect Dis 2007; 195: 202-211.

11. Lipový B1, Brychta P, Chaloupková Z, Suchánek I. Staphylococcal scalded skin syndrome in the Czech Republic: an epidemiological study. Burns 2012; 38: 296-300. 